\title{
Efficace trattamento dell'obesità ipotalamica con ossitocina e naltrexone in un ragazzo operato per craniofaringioma
}

\author{
Salvatore Cannavò ${ }^{1}$
}

Pubblicato online: 31 luglio 2018

C) Springer Nature Switzerland AG 2018

\section{Commento a:}

Oxytocin and naltrexone successfully treat hypothalamic obesity in a boy post-craniopharyngioma resection.

E.A. Hsu, J.L. Miller, F.A. Perez, C.L. Roth.

J Clin Endocrinol Metab (2018) 103:370-375

In questo recente studio Hsu e collaboratori presentano i risultati del trattamento dell'obesità ipotalamica con ossitocina e naltrexone in un ragazzo di 13 anni operato per craniofaringioma. L'obesità ipotalamica è un problema assai frequente nei pazienti, soprattutto giovani, con danno diencefalico causato direttamente da tumori o da terapie chirurgiche o radianti aggressive praticate per la loro rimozione, che non ha trovato ad oggi un trattamento realmente efficace, anche perché i meccanismi patogenetici sono solo parzialmente conosciuti. L'ossitocina è un neuropeptide ipotalamico che sembra avere un ruolo nella regolazione del bilancio energetico e che ha dimostrato proprietà anoressizzanti in animali di laboratorio, mentre il naltrexone è un antagonista oppioide che sembra potenziare gli effetti dell'ossitocina e ridurre la gratificazione provocata dall'ingestione di carboidrati.

Nell'esperienza riportata dagli autori, l'ossitocina è stata somministrata in monoterapia per via intranasale per le prime 10 settimane, a una dose iniziale di 6 UI ogni 3 giorni che è stata incrementata fino a 9 UI al giorno. A tale prima fase di trattamento è seguito un periodo di 38 settimane durante il quale l'ossitocina è stata associata al naltrexone, alla dose di $100 \mathrm{mg} /$ die. La terapia ha indotto una progressiva riduzione del BMI z score da 1,49 a 0,82 e ha ridotto l'iperfagia sia durante la prima fase di trattamento in monoterapia che durante la seconda di terapia combinata. Degno di nota è che tali risultati sono stati ottenuti attraverso un controllo corretto dell'appetito, senza la necessità di eccessivo controllo genitoriale o l'adozione di mezzi coercitivi di negazione del cibo e senza privare il ragazzo della normale ed equilibrata gratificazione indotta dall'ingestione di cibi dolci. Il trattamento si è dimostrato al contempo privo di effetti collaterali degni di nota, inclusa la temuta ritenzione idrica che l'ossitocina avrebbe potuto causare per il suo potenziale effetto antidiuretico. D'altra parte, il trattamento sostitutivo del panipopituitarismo e del diabete insipido centrale praticato in precedenza non ha subito modificazioni posologiche durante tutto il trial.

Se tale risultato fosse confermato da ulteriori studi, più ampi e controllati, si aprirebbe una concreta prospettiva di trattamento per il problema di salute più frequente e spesso drammatico, per le conseguenze sulla salute e sulla qualità di vita, riscontrato nei pazienti con tumori dell'area diencefalic,a soprattutto a seguito delle terapie chirurgiche e radianti più aggressive.

S. Cannavò

cannavos@unime.it

1 Dipartimento di Patologia Umana dell'Adulto e dell'Età Evolutiva "G. Barresi”, Università di Messina, Messina, Italia 